Glosse

\title{
Medizinische Mengenlehre
}

_ Der ältere Mensch steht derzeit im Fokus des wissenschaftlichen Interesses. Auch der Internistenkongress hat für nächstes Jahr die gesundheitlichen Belange Älterer zu einem Leitthema gemacht.

Es geht also um die Medizin für die viel zitierte 3. Lebenshälfte. Damit soll ausgedrückt werden, dass diese Hälfte an Patienten immer mehr wird und zwar zulasten der beiden anderen. Was dies für Gesellschaft und Medizin bedeutet, kann bisher keiner genau beziffern. Offen bleibt auch die Frage, wer eigentlich zur Generation „3. Lebenshälfte“ gehört bzw. wen man dazu rechnen darf, ohne politisch inkorrekt zu sein. Jedenfalls soll es die bessere Hälfte sein, und dazu hat auch die Medizin ein Stück beigetragen.
Das mit den Hälften ist übrigens in der Wissenschaft ein zunehmendes Problem. Bei immer mehr Studien profitiert nämlich nur die kleinere Hälfte von $\mathrm{Pa}$ tienten, die größere dagegen nicht. Und die 3. Lebenshälfte ist bisher in Studien so gut wie gar nicht berücksichtigt, sodass man auch gar nicht weiß, ob ein bestimmtes Medikament bei dieser Patientengruppe überhaupt wirkt. Das will man zwar jetzt ändern. Aber bis entsprechende Ergebnisse vorliegen, vergeht viel Zeit und damit auch die 3. Lebenshälfte.

Vielleicht sollte man die 3. Lebenshälfte einfach wieder abschaffen, weil wissenschaftliche Daten fehlen. Zwei Hälften reichen ja auch!

Dr. Peter Stiefelhagen
Patient unter

Antikoagulation

\section{So stoppen Sie Nasenbluten}

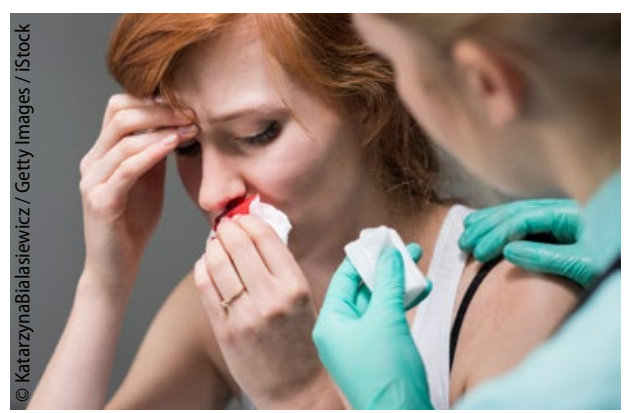

\section{Immer mehr Patienten stehen heute unter einer Theapie mit Antikoagulan- zien. Schweres Nasenbluten wird damit ein zunehmendes Problem in Kliniken und Praxen.}

Wenn sich ein Patient mit schwer beherrschbarem Nasenbluten vorstellt, sollten Sie ursächlich unter anderem an die direkten oralen Antikoagulanzien (DOAK) denken, riet Prof. Heinrich Iro, Erlangen.

Für den direkten Thrombininhibitor Dabigatran ist zwar inzwischen ein spezifisches Antidot verfügbar, aber in Situationen wie dem Hausbesuch hat man dieses wohl kaum zur Hand. Für solche Fälle empfiehlt Iro eine Maßnahme, die sich in zwei Studien als effizient erwiesen hat: Das Tamponieren der Nase mit Tranexamsäure-getränkten Schaumstoffschwämmchen. Durch die antifibrinolytische Wirkung kam es in einer Studie bei $71 \%$ der Patienten mit Epistaxis innerhalb von zehn Minuten zum Stillstand der Blutung - mit normalen Tamponaden nur bei 31\%. „Damit können Sie einigen Patienten einen operativen Eingriff ersparen“, so der Experte. - eo

- Praxis Update, München, 5./6. Mai 2017 